\section{P1-309 DIFFERENCES IN RADIOLOGICAL PATTERNS, TUMOUR CHARACTERISTICS AND DIAGNOSTIC PRECISION BETWEEN DIGITAL MAMMOGRAPHY AND SCREEN-FILM MAMMOGRAPHY IN FOUR BREAST CANCER SCREENING PROGRAMS IN SPAIN}

doi:10.1136/jech.2011.142976f.1

\begin{abstract}
${ }^{1}$ A Romero, 'L Domingo, ${ }^{*}{ }^{1} \mathrm{~F}$ Belvis, ${ }^{2} \mathrm{M}$ Sánchez, ${ }^{3} \mathrm{~J}$ Ferrer, ${ }^{4} \mathrm{D}$ Salas, ${ }^{4} \mathrm{~J}$ Ibañez, ${ }^{2} \mathrm{~A}$ Vega, ${ }^{1} \mathrm{~F}$ Ferrer, ${ }^{4}$ Mas Laso, ${ }^{1} \mathrm{~F}$ Macià, ${ }^{1} \mathrm{X}$ Castells, ${ }^{1} \mathrm{M}$ Sala. ${ }^{1}$ Department of epidemiology and evaluation, IMIM-Parc de Salut Mar, CIBERESP, Barcelona, Spain; ${ }^{2}$ General Directorate of Public Health, Department of Health and Human Services, Government of Cantabria, Santander, Spain; ${ }^{3}$ Radiology Service, Hospital de Santa Caterina, Girona, Spain; ${ }^{4}$ General Directorate Public Health \& Centre for Public Health Research, València, Spain
\end{abstract}

Introduction Since 2000 Digital Mammography (DM) has been commercially available and implemented in many breast cancer screening programs. Although available information suggests that differences, if exist, are low, complete evaluation of DM is needed to perform a more accurate balance of risks and benefits of these programs. Our purpose was to compare tumour characteristics between cancers detected with Screen-Film Mammography (SFM) and DM, and to evaluate changes on positive predictive values (PPVs), for further assessments, for invasive procedures and for different radiological patterns in recalled women.

Methods 242838 screening mammograms (171191 SFM and 71647 DM) from 103613 women aged 45-69, performed in four population-based breast cancer screening programs in Spain, were included. Tumour characteristics of each group were compared, as well as PPVs among recalled women and according radiological patterns.

Results In first and successive screenings percentages of DCIS, although not significant, were higher in DM group: $17.6 \%$ vs $13.3 \%$ $(p=0.580)$ and $19.6 \%$ vs $13.5 \%(p=0.115)$, respectively. For masses, asymmetries and calcifications PPVs were higher in DM group, being statistically significant in masses $(5.3 \%$ vs $3.9 \%$; proportion ratio: $1.3795 \%$ CI 1.08 to 1.72 ). Among cancers detected by calcifications, the percentage of DCIS was higher in DM group, being nearly significant $(60.3 \%$ vs $46.4 \%, p=0.060)$.

Conclusion PPVs were higher when DM is used, both for further assessments and for invasive procedures, with similar cancer detection rates and without statistically significant differences on tumour characteristics. Most relevant improvements on PPVs were detected for radiological patterns of masses.

\section{P1-310 STRESSFUL LIFE-EVENTS IN CHILDHOOD AND DEPRESSION IN ADULTHOOD: THE ROLE OF THE SOCIAL INTEGRATION ACCORDING TO INDIVIDUAL AND NEIGHBOURHOOD SOCIOECONOMIC STATUS}

doi:10.1136/jech.2011.142976f.2

\begin{abstract}
${ }^{1,2} \mathrm{C}$ Roustit, ${ }^{* 1,3}$ Parizot, ${ }^{1,2} \mathrm{P}$ Chauvin. ${ }^{1}$ INSERM, U707, Research Team on the Social Determinants of health and Healthcare, Paris, France; ${ }^{2}$ UPMC Univ Paris 06, Paris, France; ${ }^{3}$ Centre Maurice Halbwachs, CNRS, ENS, EHESS, Paris, France
\end{abstract}

Introduction Social integration could constitute a pathway and/ or a buffer in the association between stressful life events in childhood (SLEC) and depression in adulthood and this may differ according to the adults' socioeconomic status and neighbourhood environment. Our objective was to (1) Investigate the association between SLEC and depression in adulthood, (2) Test the mediating and/or moderating effect of social integration in the association between stressful life-events in childhood and depression in adulthood.
Methods Data. SIRS cohort study, a longitudinal epidemiological survey of 4560 adults of the Paris metropolitan area conducted since 2005. Data collection in 2005, 2007 and 2010. Outcome: New cases of depression identified by the Mini-Diag in 2010. Individual variables: SLEC, indicators of social integration (social support and social capital), socioeconomic status. Neighbourhood characteristics: socioeconomic type of the neighbourhood of residence. Statistical analysis: multilevel logistic regression and structural equations models.

Results Stressful life events (social or psychological conditions) in childhood were associated with the occurrence of a depression in 2010 (RMSEA=0.079; NFI=0.94). After including these events in a fully adjusted model, they were associated with a lack of social support in 2005, which in turn was associated with depression 5 years later, but with a different magnitude according to individuals and/or neighbourhood SES.

Conclusion Identification of pathways and buffers between stressful life events in childhood and depression in adulthood contributes to the knowledge for a comprehensive model of the intergenerational transmission of social inequalities in mental health and could guide the mental health public policies in specific sub-groups of population.

\section{P1-311 LYMPHOHEMATOPOIETIC CANCERS AND BENZENE: A POOLED ANALYSIS OF PETROLEUM WORKERS}

doi:10.1136/jech.2011.142976f.3

${ }^{1} \mathrm{~L}$ Rushton, ${ }^{*} \mathrm{D}$ Glass, ${ }^{3} \mathrm{R}$ Schnatter, ${ }^{4} \mathrm{G}$ Tang. ${ }^{1}$ Imperial College London, London, UK; ${ }^{2}$ Monash University, Melbourne, Australia; ${ }^{3}$ ExxonMobil Biomedical Sciences, Inc, New Jersey, USA; ${ }^{4}$ University of Pittsburgh, Pennsylvania, USA

Introduction There are few quantitative studies on the effect of relatively low benzene concentrations on risks of specific lymphohematopoietic cancer subtypes. Three nested case-control studies among petroleum workers in Australia, Canada and the UK have been updated and pooled to provide greater precision.

Methods To improve disease subtype classification, pathology records were obtained; two pathologists reviewed these and classified every case according to traditional and WHO classification schemes. Quantitative exposure estimates were also compared across studies to ensure that any differences in these estimates were justified. Statistical analyses employed conditional logistic regression models with flexible penalised cubic regression spline components

Results Updates identified 170 additional cases giving a total of 370 , sufficient for separate analyses by leukaemia subtypes, myelodysplastic syndrome (MDS), and myeloproliferative disease (MPD). Review of source records by pathologists resulted in changes to the underlying disease subtypes for certain leukaemia cases; pre-existing diseases such as MDS were identified; secondary polycythaemia cases were identified and excluded. Risks for acute myeloid leukaemia (AML) tended to increase as categorical benzene exposure increased when pooling the original data from the previously published studies using both the original and revised exposure assessment. Dose-response results from the updated pooled data for MDS, MPD, AML and chronic myeloid leukaemia, and chronic lymphoid leukaemia will be presented from the updated dataset.

Conclusions This pooled study benefited from careful reconsideration of benzene exposure estimates and disease classification procedures, improving the precision of risk estimates of benzene exposure for leukaemia and other disease subtypes. 